\title{
Optimizing Application of Image Analysis Tools to Determine Physical Characteristics of Fine Roots
}

\author{
Arthur Genis*, Dan G. BlumberG* and Jiftah Ben-ASher*** \\ * Department of Geography and Environmental Development, Ben-Gurion University \\ of the Negev, P.O. Box 653, 84105 Beer-Sheva, Israel \\ ** Jacob Blaustein Institute for Desert Research, Ben-Gurion University of the Negev, \\ Sede Boqer Campus, 84990 Israel
}

(Received May 10, 2005)

\begin{abstract}
To understand the vital role of roots in plant growth, it is essential to know the dynamics and distribution of the plant root system. Research in this area has been limited severely by labor intensive techniques used for determination of physical characteristics of roots, especially fine roots. Nowadays, with development of various image analysis applications, it is possible to use methods that speed the process of fine root quantifying and, therefore, allow many more samples to be collected and processed for study. The accuracy of root measurements and time required greatly depend on the capabilities of the image acquisition equipment and the employed root preparation technique. At the same time, the optimal use of image analysis software can improve dramatically both precision and measuring speed. Our objective was to examine some fine root measuring issues common to most image analysis applications. These problems include choosing the proper image resolution, determining the appropriate threshold range and image parameters to measure, as well as reducing the impact of non-root objects on measurement accuracy. Using a numerical example, we describe techniques that can be used to minimize these problems and optimize the precision and accuracy of measurements. Our results suggest that optimal scanning resolution can be determined based on prior determination of root diameter and the minimum root diameter in an image must contain no less than three pixels. We also found that variation in measured root length can by minimized by determining threshold ranges. By applying numerical filters to images, we were able to decrease background noise ; however determination of the optimal filter ranges needed to be performed individually for each application and depend on the size of scanned roots and image resolution. The combined effect of using optimal image resolution, threshold ranges, and numeric filters can increase the efficiency of fine root image analysis in terms of precision and speed.
\end{abstract}

Keywords : background noise, fine roots, identification threshold, image analysis, spatial resolution

\section{INTRODUCTION}

Manual root measurement is time consuming, tedious, and labor-intensive work (Box, 1996). Accordingly, manual measurements of fine and very fine roots create even more difficulties and are not always possible. At the same time, image analysis of fine roots is fast and, relative to manual

Corresponding author: Arthur Genis, fax : +972-8-991-7019

e-mail : argenis@bgu.ac.il 
techniques, has a low labor requirement. Recently, root research became more oriented to analysis of transport problems; root length and root surface have replaced root dry weight as the root parameter of primary interest (Van Noordwijk, 1987). While the root parameters may be estimated by manual root tracing in the image, the tool that speeds image analysis is automatic computer analysis (Ingram and Leers, 2001). Although such automation can resolve greatly the issue of fast image capturing but time consuming and expensive manual analysis of root images (Jose et al., 2001), there are some important questions to consider. These include: the right choice of image resolution and intensity threshold; minimization of fragmentation effect (artificial root cuts in the image) and background noise.

Spatial resolution for scanning very fine roots is time and hardware demanding. At the same time there is usually a compromise between minimal required resolution and time of root image acquisition. Conversely, intensity threshold range is not a time limited factor, and its selection is completely subjective. However, the choice of intensity threshold has a great impact on the determination of root parameters, and its incorrect application leads to a dramatic increase in error. The impact of non-root objects, or background noise, is usually resolved by various image enhancement techniques. However, using enhancement techniques on fine roots images can result in distortion of image data, and consequently incorrect root measurements.

Specific details and application of commercially available root scanning systems have been discussed by many authors (Bouma et al., 2000; Ortiz-Ribbing and Eastburn, 2003; Bauhus and Messier, 1999; Zobel, 2003). Our objectives were to examine root measuring issues common to most automated image analysis applications by determining whether (1) optimal resolution for image analysis can be selected based on root diameter; (2) calculating root length as a function of applied threshold range increases objectivity of root measurements and decreases variation; and (3) numerical filters can be used to decrease background noise in images.

\section{THEORY}

\section{Determination of root identification threshold}

A root image is a digital representation of real roots. Created with a digital camera or a scanner it is used to make measurements. The image is represented by a grid of pixels, for which in an eight bit monochrome image a value between 0 (black) and 255 (white), called a digital number (DN), is assigned. Gray-level or intensity thresholding is a segmentation of an input image into two classes-one for those pixels having values below a defined gray level and one for those above this value (Lillesand and Kiefer, 1987). For the situation when roots are darker than the background against which they were scanned, it is possible to apply an intensity threshold range within which all the pixels are darker than the background, and outside which all the pixels are lighter than roots. In this case the lower limit of the threshold range is always 0 and the upper limit directly determines all measured object parameters, so its correct selection is crucial. This is why we call the upper limit of the threshold range a 'root identification threshold' $T h$. Increasing $T h$ increases the number of corresponding pixels used in the measurements, and consequently increases the calculated values of root parameters (e.g. length and surface area). For example, applying Th of 130, 160,190 , or 220 to the same root object (Fig. 1), results in the object's area $S$ to consist of 58, 106, 155, or 247 pixels and the object's perimeter $P$ to be 60,74, 78, and 92 pixels. A 70\% increase in $T h$ can result in increase in $S$ of over $300 \%$ and an increase in $P$ of approximately $53 \%$. This phenomenon can be explained by the generally elongated shape of roots. The more elongated the shape of an image object, the less impact $T h$ has on $P$. This suggests when optimal $T h$ can not be selected, $P$ should be used as a principal parameter for image analysis of roots to reduce error.

It is, however, possible to obtain an optimal $T h$ for different image analysis applications by testing applications for sensitivity using the derivative $d L / d T h$, where $L$ is the number of pixels 


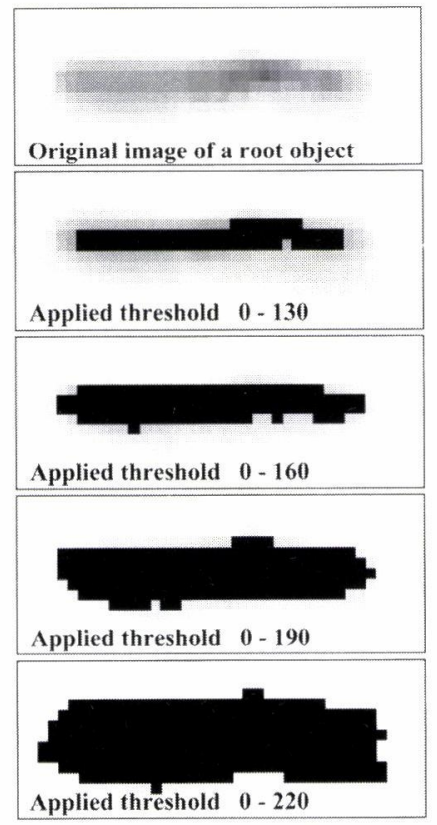

Fig. 1 Effect of the applied root identification threshold $T h$ on the resulting object's shape and size. Shown is the same root image with different threshold ranges applied.

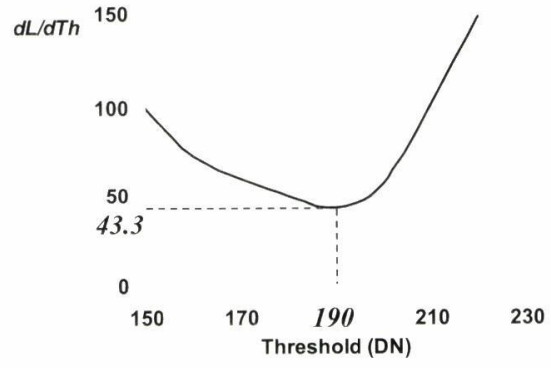

Fig. 2 Effect of the selected root identification threshold $T h$ on the derivative $d L / d T h$. The minimum variation of determined root object length $L$ corresponds to $T h=$ 190.

representing root length, and $T h$ is the applied root identification threshold. For example, applying Th of 150-220 to image objects with $L$ of 7600 pixels, results in a $d L / d T h$ of 43.3 (Fig. 2).

This corresponds to a $T h$ of 190 being associated with a minimal change in $L$. In other words, moving around this value of threshold leads neither to deduction of "darker" pixels (with less DN) of the root representation, nor to capture of "brighter" pixels (with higher DN) of the background. For the given application, this Th can be considered as the best possible estimate for such root parameters as length, diameter and surface area.

Determination of optimal resolution and pixel size

Another factor affecting precision of measurements is spatial resolution of the image, which is the ratio of the number of pixels comprising the image versus the actual subject size. Spatial resolution of scanned images is determined by the number of pixels per $\mathrm{mm}\left(\mathrm{p} \mathrm{mm}^{-1}\right)$ and enlargement settings used when scanning the image, while some scanning software employs the American units for resolution dpi (dots per inch). Unlike intensity resolution (which determines how fine a difference in light intensity can be detected), spatial resolution cannot be changed after image acquisition, so its value is selected a priori. The higher the spatial resolution of a root image, the 'finer' the measurements, i.e. increasing image resolution increases precision because measurements are made from pixel to pixel. For example, a root image object at $8 \mathrm{p} \mathrm{mm}^{-1}$ resolution is composed of much fewer pixels than the same root image object at $24 \mathrm{p} \mathrm{mm}^{-1}$ (Fig. 3).

The pixel size is inversely proportional to the resolution and, if no software enlargement or hardware magnification is involved, equals

$$
p=\frac{1}{R}
$$




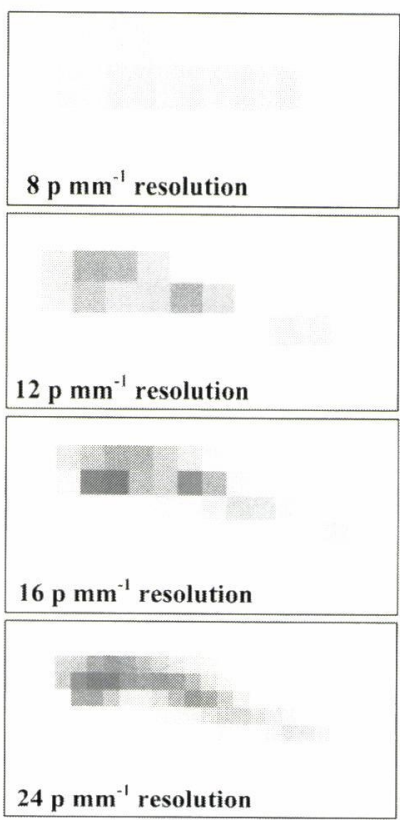

Fig. 3 Effect of scanning resolution $R$ on the root image.

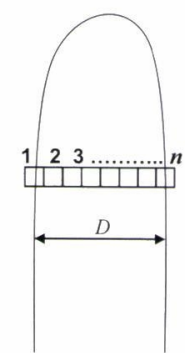

Fig. 4 Schematic of an actual root. Root diameter $D$ expressed by $n$ associated pixels in the image.

where: $\quad p$ is size of a pixel $(\mathrm{mm})$

$R$ is spatial resolution ( $\mathrm{p} \mathrm{mm}^{-1}$ )

Increased resolution also increases image acquisition time. To optimize spatial resolution for image acquisition, estimates of root width and its variation need to be considered along with time and hardware constraints. The mean width of the root and the standard deviation are two ways to characterize optimal spatial resolution. For example, if the highest possible contrast is applied to the image, i.e. roots are black $(\mathrm{DN}=0)$ and the background is white $(\mathrm{DN}=255)$, and the root width is $n$ pixels (Fig. 4), then we can assume that the two extreme pixels \#1 and $\# n$ are half white and half black with $\mathrm{DN}=255 / 2$.

Thus the mean DN is

$$
\overline{\mathrm{DN}}=\frac{1}{n} \sum_{i=1}^{n} D N_{i}=\frac{1}{n}(0.5 \times 255+0 \ldots+0+0.5 \times 255)=\frac{255}{n}
$$

Then the standard deviation (SD) is

$$
\mathrm{SD}=\left[\frac{1}{n-1} \sum_{i=1}^{n}\left(\mathrm{DN}_{i}-\overline{\mathrm{DN}}\right)^{2}\right]^{0.5}
$$

In equations (2) and (3):

$$
n=D \cdot R
$$

where: $n$ is the number of pixels representing root diameter

$D$ is root diameter $(\mathrm{mm})$

Using large roots $(D=0.2 \mathrm{~mm})$ and small roots $(D=0.1 \mathrm{~mm})$ as an example, we see decrease of $\mathrm{SD}$ as $R$ increases (Fig. 5).

At any given $R$, variation in $\mathrm{SD}$ is increased with decreasing of the root size. To select a rea- 


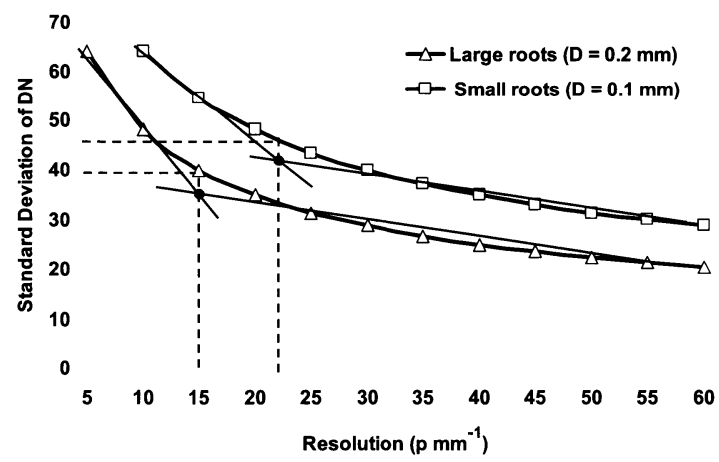

Fig. 5 Effect of image resolution $R$ on standard deviation SD determined by digital numbers DN of pixels within the root diameter $D$.

sonable resolution for root scanning we can describe lines in the graph in Fig. 5 by two linear steps or sections: 1) a steep slope with a large reduction of $\mathrm{SD}$ in response to a small change in $R$; and 2 ) a moderate slope with a small reduction of SD in response to a large change in $R$. Scanning time decreases with decreasing $R$, therefore we subjectively selected the intersection between the two steps or sections of the line as an optimal point at which reduced resolution is associated with minimal increase of SD. Then each section is replaced by a linear trendline, so that the best fitting is achieved. The minimum required resolution can then be calculated as follows:

The equation for the steep slope section of the line is

$$
\mathrm{SD}_{1}=a_{1} R+b_{1}
$$

And the equation for the moderate slope section of the line is

$$
\mathrm{SD}_{2}=a_{2} R+b_{2}
$$

where $a_{1}$ and $a_{2}$ are the slopes, $b_{1}$ and $b_{2}$ are the intercepts.

The two sections of the line intersect where $\mathrm{SD}_{1}=\mathrm{SD}_{2}$, so that

$$
a_{1} R+b_{1}=a_{2} R+b_{2}
$$

Therefore the minimum required resolution is

$$
R_{\min }=\left(b_{2}-b_{1}\right) /\left(a_{1}-a_{2}\right)
$$

Using this set of equations we extracted two linear steps (trendlines) for each of the lines in Fig. 5 and determined that $R_{\min }=22.0 \mathrm{p} \mathrm{mm}^{-1}$ for $0.1 \mathrm{~mm}$ roots and $R_{\min }=14.9 \mathrm{p} \mathrm{mm}^{-1}$ for $0.2 \mathrm{~mm}$ roots (Table 1). For each linear trendline its slope ' $a$ ' and intercept ' $b$ ' are determined automatically by Microsoft Excel.

Obtained resolutions produce standard deviations $\mathrm{SD}=46$ for small roots and $\mathrm{SD}=40$ for large roots, while the relative errors are $18 \%$ and $16 \%$ respectively. That is for the larger roots we can reduce scanning resolution (and, consequently, scanning time) without loosing observational precision. Table 1 demonstrates that the linear approximation yielded high correlation coefficients $r^{2}$ and reasonable minimum required resolutions, while $n>2$. Since in reality the number of pixels is a natural number, this suggests that the actual $n$ can not be less than three $(n \geq 3)$. In this case at least one pixel is completely inside the diameter of a root object (Fig. 4), which sustains the object's integrity.

Non-root objects and application of a filter

Total $S$ and $P$ of all objects recognized by an application are initially measured in pixels. However, the number of recognized objects is usually much higher than number of roots in the 
A. GENIS ET AL.

Table 1. Linear parameters of standard deviation (SD) in Fig. 5 and equations (5)-(8).

\begin{tabular}{ccccccccccc}
\hline \multirow{2}{D}{} & & & & & & & \multicolumn{2}{c}{ Calculated } & \multicolumn{2}{c}{ Practical } \\
\cline { 6 - 10 } & $a_{1}$ & $b_{1}$ & $r_{1}{ }^{2}$ & $a_{2}$ & $b_{2}$ & $r_{2}{ }^{2}$ & $\begin{array}{c}R_{\min } \\
(\mathrm{p} \mathrm{mm})\end{array}$ & $n$ & $\begin{array}{c}R_{\min } \\
\left(\mathrm{p} \mathrm{mm}^{-1}\right)\end{array}$ & $n$ \\
\hline 0.1 & -1.91 & 83.1 & 0.99 & -0.34 & 48.3 & 0.98 & 22.0 & 2.2 & 30 & 3 \\
0.2 & -3.18 & 79.4 & 0.99 & -0.27 & 36.1 & 0.98 & 14.9 & 3.0 & 15 & 3 \\
\hline
\end{tabular}

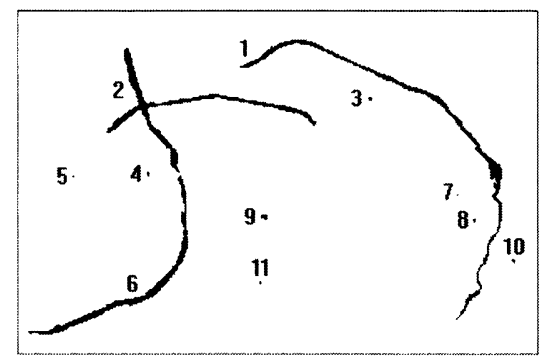

Fig. 6 Fragment of a root image with 11 objects comprising both root objects and the background noise.

image. Some objects are very big-hundreds of pixels in size, but most of them hardly exceed few pixels. This discrepancy takes place because after intensity thresholding, some single roots, as a result of either their poor contact with the surface of the scanner or their partial discoloration, appear as multiple big objects (fragmentation), while small non-root objects-usually grains of soil left after root-washing-are seen as multiple small objects (background noise). For example a scan of two root pieces can detect many more image objects (Fig. 6). Eleven image objects are initially counted by the program although in reality only two are roots. Object 1 is a single root, objects 2 and 6 are a single root that has been fragmented by intensity thresholding, and other objects are background noise.

Even after applying certain image enhancement techniques (adjusting brightness and contrast, edge detecting, convolution filtering etc.) to improve the intensity resolution, it is impossible to eliminate the fragmentation and background noise completely. To minimize their effect on measurements, we suggest application of an object filter. We presume that objects are not fragments of roots, if their area $S^{\prime}$ is less than certain arbitrary value $Z$ for a given resolution, and therefore these objects must be filtered out. An example of such a filter for the image in Fig. 6 (with resolution $24 \mathrm{p} \mathrm{mm}^{-1}$ and $Z=30$ pixels) is shown in Table 2 .

In Table 2 only area and perimeter of objects 1,2 and 6 are taken into account to calculate the total area and perimeter of roots in the image. In this example only three objects out of 11 were recognized as roots. The filtration process did not affect the total area of all objects by more than $2 \%$. The difference for recalculated total perimeter is $3 \%$. At the same time, the impact of segmentation on the final results was minimal: the total area of all objects in Fig. 6 consists of 1723 pixels, while the total loss for segmentation does not exceed 4-5 pixels. Nevertheless, an object filter must be selected so that its size is less than any possible size of a root segment. For fine root images with high resolution and large image area, as the fragmentation and background noise increase, the role of filtering also increases.

\section{Calculation of basic root parameters}

The fundamental root characteristics are its length and surface area. For simplicity we consider roots as cylinders with the diameter $D$ and the length $L$, subsequently we can consider projections of roots in the image as rectangles with the width $D$ and length $L$. Then the perimeter $P$ of an object in the image can be expressed as: 


\section{IMAGE ANALYSIS OF FINE ROOTS}

Table 2. An example of filtering root objects in Fig. 6, and calculating their total area and perimeter.

\begin{tabular}{|c|c|c|c|c|c|}
\hline \multirow{3}{*}{ Object \# } & \multicolumn{2}{|c|}{ Measured } & \multicolumn{2}{|c|}{ Filtered } & \multirow{3}{*}{$\begin{array}{l}\text { Object } \\
\text { counter }\end{array}$} \\
\hline & Area $S$ & Perimeter $P$ & Area $S^{\prime}>Z=30$ & Perimeter $P^{\prime}$ & \\
\hline & \multicolumn{4}{|c|}{ pixels } & \\
\hline 1 & 654 & 636 & 654 & 636 & 1 \\
\hline 2 & 610 & 460 & 610 & 460 & 1 \\
\hline 3 & 2 & 5 & 0 & 0 & \\
\hline 4 & 5 & 8 & 0 & 0 & \\
\hline 5 & 1 & 3 & 0 & 0 & \\
\hline 6 & 459 & 350 & 459 & 350 & 1 \\
\hline 7 & 1 & 3 & 0 & 0 & \\
\hline 8 & 1 & 3 & 0 & 0 & \\
\hline 9 & 10 & 11 & 0 & 0 & \\
\hline 10 & 3 & 6 & 0 & 0 & \\
\hline 11 & 2 & 5 & 0 & 0 & \\
\hline Total & 1,748 & 1,490 & 1,723 & 1,446 & 3 \\
\hline
\end{tabular}

$$
P=2 D+2 L
$$

while its area is:

$$
S=D \cdot L
$$

Since the diameter value of a root is negligible comparative to its length, for the first approximation the length can be calculated as:

$$
L=P / 2
$$

If $P$ is in pixels, then to transfer to millimeters or centimeters, $L$ must be multiplied by pixel size $p$.

Then $D$ can be recalculated as:

$$
D=\frac{S}{L} \cong \frac{2 S}{P}
$$

The root surface area $S^{*}$ can be calculated as:

$$
S^{*}=\pi D L \cong \pi \cdot \frac{2 S}{P} \cdot \frac{P}{2} \cong \pi S
$$

If $S$ measures in pixels, then to transfer to square millimeters or centimeters, $S^{*}$ must be multiplied by $p^{2}$. Calculated $\mathrm{S}^{*}$ is directly proportional to determined $S$, while calculated $L$ is directly proportional to determined $P$. Equations (11) and (13) allowed us to calculate root length and surface area, which are essential indicators for potential uptake of water and nutrients.

\section{MATERIALS AND METHODS}

In this section we present root image analysis technique and associated calculations used to test the theoretical findings. The employed real root sample preparation method was purposely not optimized by any image contrast enhancement such as root staining in order to provide objectivity to the test. The intention was 1) to exclude any possible distortion of image of very fine roots; 2) to maximize uniformity of processing the image by any image processing application; 3 ) to reduce as much as possible time of data acquisition. As Jensen (1996) states, there is no such thing as the ideal or best image enhancement because the results are ultimately evaluated by humans, who make 
subjective judgments as to whether a given enhancement is useful.

Using samples of known length and diameter to characterize root properties

We tested various image analysis applications for their ability to determine root length and surface area. The test was performed on artificial roots, presented by wire with known length and diameter. The artificial root diameter was $D=0.22 \mathrm{~mm}$. For one scan, $10 \mathrm{~cm}$ of artificial roots were cut into seven pieces of differing lengths and shapes, and dispersed across the imaging area with no overlapping between pieces (Fig. 7a). For the second scan, $50 \mathrm{~cm}$ of artificial roots were cut into 20 pieces of differing lengths and shapes, and randomly dispersed across the imaging area with some overlapping between pieces (Fig. 7b). The images were scanned against white background (Whatman filter paper \# 40, $\phi=11.0 \mathrm{~cm}$ ) by a standard flatbed scanner EPSON Perfection 1250 (SEIKO EPSON CO., Japan), using Epson twain v.5.53E program for image capture provided with the scanner. Both images were saved as JPEG format 8-bit/pixel (256 grayscale) monochrome images with standard (not progressive) encoding and $90 \%$ compression level. According to equation (4), the calculated minimum resolution (when $n=3$ ) is $R_{m i n}=13.6 \mathrm{p} \mathrm{mm}^{-1}$, so the actual resolution used was $R=16 \mathrm{p} \mathrm{mm}^{-1}$ ( $400 \mathrm{dpi}$ ). The scanning equipment was calibrated by drawing a 50 $\mathrm{mm}$ line on the paper circle, scanning it and measuring its length in pixels. The verified pixel size was $p=0.063 \pm 0.0005 \mathrm{~mm}$.

SigmaScan ${ }^{\mathrm{R}}$ Pro 5 (SPSS Inc., USA) and ImageTool 3.0 (UTHSCSA, USA) were used for image processing and analysis. $T h=190$ was applied as described in previous section. The filter $Z=30$ pixels was selected for these images because under given conditions (scanning resolution and applied root identification threshold) no root objects are smaller than $Z$, while no background noise objects exceed $Z$.

Analysis of actual roots

Four-week old Lettuce (Lactuca sativa L. cv. "Noga") plants grown in a greenhouse in pots were used for the analysis. Root samples were collected by means of a thin-walled metal cylinder plunged vertically into the soil to the depth of $10 \mathrm{~cm}$, and each sample represents soil volume of $2.7 \mathrm{~cm}^{3}$. Roots were separated by washing from sand, used as growing media, and then filtered through filter paper (Whatman \# 40, $\phi=11.0 \mathrm{~cm}$ ) in the Buchner funnel with a perforated filter plate. While filtering, roots were untangled and evenly spread on the surface of the filter paper circle using a plastic spatula to minimize overlapping. After drying roots were scanned against white background of the filter paper using the same scanner and parameters as for artificial roots. For the image analysis of actual roots, two different samples were considered: one containing tap and lateral roots, and the other containing only lateral roots. Images of these roots are presented in Fig. 8 .

In Fig. 8a roots have various diameters and relatively high density, while in Fig. 8b roots have nearly constant diameter of $0.1 \mathrm{~mm}$ and very low density. As the thinnest roots in both cases had

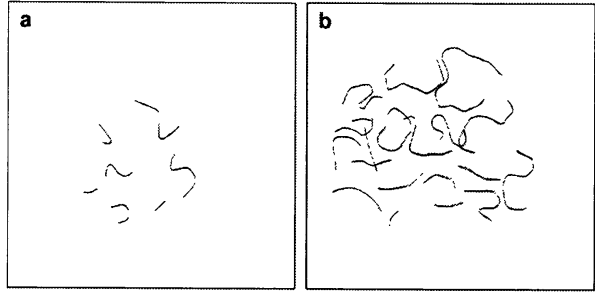

Fig. 7 a. Scanned image of artificial roots with total length $L=10 \mathrm{~cm}$. b. Scanned image of artificial roots with total length $L=50$ $\mathrm{cm}$.

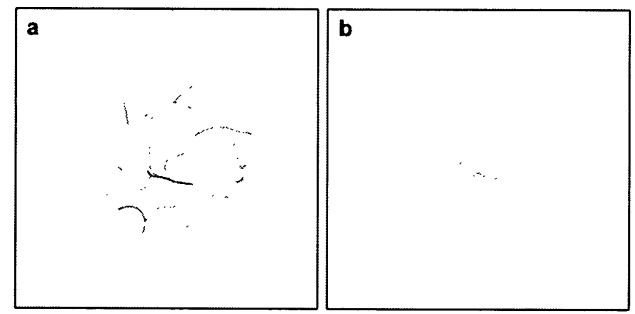

Fig. 8 a. Scanned image of actual tap and lateral roots. b. Scanned image of actual lateral roots. 


\section{IMAGE ANALYSIS OF FINE ROOTS}

diameter of approximately $0.1 \mathrm{~mm}$, to maintain $n \geq 3$ and according to Table 1 , the scanning resolution in both cases was chosen $R=24 \mathrm{p} \mathrm{mm}^{-1}$ ( $600 \mathrm{dpi}$ ). Since roots are darker than the white background, applying the root identification threshold $T h=190$, as described in the theory section, highlighted all the roots and defined them as 'objects'. Like with artificial roots, the filter $Z$ of 30 pixels was applied.

For actual roots, to validate the results obtained by automatic image analysis, manual root tracing was carried out on the same two images. The manual tracing was performed using capabilities of ERDAS IMAGINE V8.6 (Leica Geosystems, USA) and was accompanied by constant monitoring of real samples. Although a very time-consuming, this method is the most precise available, because it involves pixel-to-pixel measurements which allowed using the results as a reference.

\section{RESULTS AND DISCUSSION}

\section{Artificial roots}

Using equation (11) and (13) for the $10 \mathrm{~cm}$ and $50 \mathrm{~cm}$ artificial roots (Fig. 7a and b) the total length was measured $11.2 \mathrm{~cm}$ and $55.5 \mathrm{~cm}$ respectively. Hence, the calculated length overestimated actual length by $12 \%$ and $11 \%$. We attribute such overestimation of artificial root length to the overestimated total perimeter $P$ due to shadowing (Bouma et al., 2000), as artificial roots are less flat and flexible than real roots.

As actual surface area of the artificial roots is $0.7 \mathrm{~cm}^{2}$ and $3.5 \mathrm{~cm}^{2}$, the determined surface area was $0.6 \mathrm{~cm}^{2}$ and $3.2 \mathrm{~cm}^{2}$ respectively. Hence, the calculated surface area underestimated actual surface area by $14 \%$ and $8 \%$. According to our observations, this underestimation is also caused by certain inflexibility of artificial roots comparative to real roots, which prevented them from complete contact with the glass surface of the scanner.

\section{Actual roots}

While the real number of root objects in the image presented in Fig. 8a is 12, an image analysis application recognized 266 objects consisting of pixels with DN less than 190 . Total area of all these objects was determined as $S=27065$ pixels; total perimeter $P=15334$ pixels. After application of the filter the number of objects was reduced to 21 . For these 21 objects total area is $S^{\prime}$ $=26181$ pixels, and total perimeter is $P^{\prime}=13830$ pixels. Thus, calculations revealed decrease by $3 \%$ of total area of all objects after the filter application, while total perimeter of the objects after filtering was reduced by $10 \%$. The number of identified root objects is greater than real number of roots because of threshold fragmentation. The pixel size, according to equation (1), was calculated as $p=1 / R=1 / 24=0.042 \mathrm{~mm}$. According to equation (11), the total root length is $L=$ $13830 / 2=6915$ pixels, that is $L=29.0 \mathrm{~cm}$. According to equation (13), the total root surface area is $S^{*}=\pi \cdot S=3.14 \cdot 26181=82208$ pixels, that is $S^{*}=1.45 \mathrm{~cm}^{2}$.

For the image presented in Fig. $8 \mathrm{~b}$ the real number of root objects is four, recognized was 247 objects and filtering reduced this number to six. For this image, using the same equations and principals, we calculated the total root length $L=4.6 \mathrm{~cm}$ and the total root surface area $S^{*}=0.19 \mathrm{~cm}^{2}$. Manual root tracing, using ERDAS, produced the total root length: $L=29.4 \mathrm{~cm}$ (Fig. 8a) and $L=$ $4.9 \mathrm{~cm}$ (Fig. 8b), corresponding to errors of $1.3 \%$ and $6 \%$ towards underestimation by automation. This probably indicates increase of error in response to decrease of average diameter of roots in the sample.

However, the error in both cases is relatively low, if one takes into account the following:

- no sample or image enhancement was employed;

- the image analysis process was fully automated with data acquisition time less then $30 \mathrm{~s}$ per image;

- the increase of number of root objects with consequential increase of object density and overlapping, did not lead to increase of error. 


\section{A. GENIS ET AL.}

Bouma et al. (2000) found that the choice of software is much less important than a proper scanning protocol used with respect to staining period, sample density, scanning resolution and threshold range. They suggest for the most sensitive parameters a root staining period of $24 \mathrm{~h}$ and a resolution of $16 \mathrm{p} \mathrm{mm}^{-1}$ should be used. They also indicate as optimal Th of 130-210 for Delta-T Scan and 170-220+ Automatic for WinRHIZO, which corresponds with our findings presented in Fig. 2. At the same time in the present study we intentionally excluded from observation the root staining technique as well as any image enhancements (Costa et al., 2001). As root preparation and scanning protocols vary widely between laboratories (Bouma et al., 2000), our results indicate that the use of optimal parameters for root image analysis leads to adequate results even without a significant sample preparation.

Our error analysis shows that the larger is the number of pixels per root diameter, the less is standard deviation. In our analysis we concluded that the minimum theoretical number is three, which insures that at least one pixel is completely inside the diameter of a root object or, otherwise, the root object cannot be determined. This finding corresponds to the statement of Zobel (2003) that pixel size needs to be less than one-third the diameter of the smallest object being imaged.

We also conclude that knowing in advance the appropriate scanning resolution $R$ benefits both the precision and speed of image acquisition, while applying the correct root identification threshold $T h$ leads to optimal data processing and truthful results. In case of fine and very fine root analysis, application of numerical filter can be very important. Depending on amount of the background noise, image density and image size, filtering can significantly reduce the error when calculating root parameters. The presented analysis in combination with a reliable root automation technique may help objectively determine fine roots characteristics and speed up root studies in general.

\section{REFERENCES}

Bauhus, J., Messier, C. 1999. Evaluation of fine root length and diameter measurements obtained using RHIZO image analysis. Agron. J. 91: 142-147.

Bouma, T. J., Nielsen, K. L., Koutstall, B. 2000. Sample preparation and scanning protocol for computerized analysis of root length and diameter. Plant Soil 218: 185-196.

Box, J. E. 1996. Modern Methods for Root Investigations. In "Plant Roots: The Hidden Half" (ed. by Y. Waisel, A. Eshel, U. Kafkafi), Marcel Dekker, New York, p 193-237.

Costa, C., Dwyer, L. M., Hamel, C., Muamba, D. F., Wang, W. L., Nantais, L., Smith, D. L. $2001 . \quad$ Root contrast enhancement for measurement with optical scanner-based image analysis. Can. J. Bot. 79: 23-29.

Ingram, K. T., Leers, G. A. 2001. Software for measuring root characters from digital images. Agron. J. 93: $918-922$.

Jensen, J. R. 1996. Introductory Digital Image Processing: A Remote Sensing Perspective. Prentice Hall, NJ, pp 318.

Jose, S., Gillespie, A. R., Seifert, J. R., Pope, P. E. 2001. Comparison of minirhizotron and soil core methods for quantifying root biomass in a temperate alley cropping system. Agrofor. Syst. 52: 161-168.

Lillesand, T. M., Kiefer, R. W. 1987. Remote Sensing and Image Interpretation. John Wiley \& Sons, NY, pp 721.

Ortiz-Ribbing, L. M., Eastburn, D. M. 2003. Evaluation of digital image acquisition methods for determining soybean root characteristics. Online. Crop Management, doi: 10.1094/CM-2003-0702-01-RS.

Van Noordwijk, M. 1987. Methods for quantification of root distribution pattern and root dynamics in the field. Proc. 20th Colloq. Intern. Potash Inst., Boden bei Wien, p 263-281.

Zobel, R. W. 2003. Sensitivity analysis of computer-based diameter measurement from digital images. Crop Sci. 43: 583-591. 